\title{
INTEGRATION OF SIMPLICIAL FORMS AND DELIGNE COHOMOLOGY
}

\author{
JOHAN L. DUPONT and RUNE LJUNGMANN*
}

\begin{abstract}
We present two approaches to constructing an integration map along the fiber for smooth Deligne cohomology. The first is defined in the simplicial model, where a class in Deligne cohomology is represented by a simplicial form, and the second in a related but more combinatorial model.
\end{abstract}

\section{Introduction}

For the construction of invariants for families of bundles, integration along the fiber is usually applied in order to obtain forms defined on the parameter space. In the case of families of bundles with connection the classical ChernWeil theory gives rise to invariants living in smooth Deligne cohomology, and hence a notion of integration along the fiber is needed in this setting (see e.g. Freed [6] or Dupont-Kamber [5]). In this paper, we introduce two different constructions of this map. The first one is defined in the simplicial model for smooth Deligne cohomology introduced in [5], where a class in the smooth Deligne cohomology $H_{\mathscr{D}}^{l+1}(Z, Z)$ is represented by a simplicial form $\omega \in \Omega^{l}(|N \mathcal{U}|)$, for $\mathcal{U}$ a covering of $Z$. This version of the integration map is needed in [5] for the construction of invariants for families of foliated bundles but it was defined there only in the case of a product bundle. In general we prove that:

THeOREM 1.1. Given a fiber bundle $\pi: Y \rightarrow Z$ with compact, oriented $n$-dimensional fibers and suitable coverings $\mathscr{V}$ and $\mathscr{U}$ of $Y$ and $Z$ respectively. Then there is a map

$$
\int_{[Y / Z]}: \Omega^{*+n}(|N \mathscr{V}|) \rightarrow \Omega^{*}(|N \mathscr{U}|)
$$

\footnotetext{
*Work supported in part by the Danish Natural Science Research Council and the European Union Network EDGE.

Received May 25, 2004.
} 
It satisfies a Stokes' formula

$$
\int_{[Y / Z]} d \omega=\int_{[\partial Y / Z]} \omega+(-1)^{n} d \int_{[Y / Z]} \omega,
$$

and thus if $\partial Y=\emptyset$ induces a map

$$
\pi_{!}: H_{\mathscr{D}}^{*+n}(Y, \mathrm{Z}) \rightarrow H_{\mathscr{D}}^{*}(Z, \mathrm{Z})
$$

in smooth Deligne cohomology independent of all choices and compatible with usual integration along the fibers.

In the course of the proof of this theorem we make a second construction of the integration map defined in a more combinatorial model where the cohomology classes are represented by simplicial forms living in the "triangulated nerve' $|N K|$ associated to a triangulation $|K| \rightarrow|L|$ of the bundle. This allows us to state the following useful theorem in the case where the fiber has boundary:

Theorem 1.2. Assume that $\partial Y \neq \emptyset$. Then for a form $\omega \in \Omega^{*+n}(|N \mathscr{V}|)$ representing an element in smooth Deligne cohomology, the form

$$
\int_{K / L} \omega \in \Omega^{*}(|N L|) / d \Omega^{*-1}(|N L|)
$$

depends only on the triangulation of $\partial Y \rightarrow Z$.

There are other approaches to the subject in the literature. In Hopkins-Singer [8], a cochain model for the Cheeger-Simons differential characters is given and an integration map is constructed by embedding the bundle in a larger trivial one. However for the applications in [5] one needs a construction involving local data as in theorem 1.1. In the Čech-de Rham model, Gomi-Terashima [7] have introduced a combinatorial formula that uses a triangulation of the fiber. Unfortunately their formula is given for product bundles only, and it is not immediately clear how to generalise it to the case of a general fiber bundle.

Apart from giving the applications in [5] our integration map is well adapted to the product structure in Deligne cohomology. This answers a question asked us by Ulrich Bunke. Thus we hope to demonstrate that the approach with simplicial forms is a convenient and natural generalisation of the usual integration map.

We will start by giving a short description of smooth Deligne cohomology both in the usual Čech-de Rham model and in the simplicial model introduced in [5] in $\S 2$. In $\S 3$, we introduce the concept of prism complexes which is a generalisation of simplicial sets well suited for fiber bundles. It will provide 
a convenient framework for the constructions in $\S \S 4-5$. In $\S 4$, we construct an integration map in the simplicial model by choosing suitable coverings of the fiber bundle and a set of partitions of unity. In $\$ 5$, we introduce a more combinatorial model closely related to the simplicial approach. By using an Alexander-Whitney type map, we then give a combinatorial integration formula. We show that the two approaches induce the same map in smooth Deligne cohomology and at the same time we end the proof of theorems 1.1 and 1.2. Finally in $\$ 6$ we define the product in Deligne cohomology using simplicial forms and show that the integration map is well behaved with respect to this product.

AcKNOwLedgements. The authors would like to thank Ulrich Bunke, Marcel Bökstedt and Franz Kamber for useful discussions during the preparation of this paper.

\section{Smooth Deligne cohomology}

Here follows a short introduction to smooth Deligne cohomology. The Deligne cohomology groups are usually constructed as the hypercohomology of a certain sequence of sheaves. We will however restrict ourselves to the corresponding concrete Čech description along the lines of [5]. For a more comprehensive exposition see Brylinski [1].

Let $Z$ be a smooth manifold of dimension $m$ and let $\mathscr{U}=\left\{U_{i}\right\}_{i \in I}$ be a 'good' open cover of $Z$. (That is every non-empty intersection of sets from the covering is contractible).

Let $\check{\Omega}^{p, q}(\mathscr{U})=\check{C}^{p}\left(\mathscr{U}, \underline{\Omega}^{q}\right)$ be the ordinary $\check{C}$ ech-de Rham complex and let $\check{\Omega}^{*}(\mathscr{U})$ denote the corresponding total complex with total differential $D=$ $\delta+(-1)^{p} d$ on $\check{\Omega}^{p, q}(\mathscr{U})$. It is well-known that the chain map

$$
\varepsilon^{*}: \Omega^{q}(Z) \rightarrow \check{\Omega}^{0, q}(\mathscr{U}),
$$

induced by the natural map $\varepsilon: \sqcup U_{i} \rightarrow Z$ gives an isomorphism

$$
H_{\mathrm{dR}}^{*}(Z) \rightarrow H^{*}\left(\check{\Omega}^{*}(\mathscr{U})\right)
$$

in cohomology. We also have an inclusion of the ordinary Čech-complex with integer coefficients

$$
\check{C}^{p}(\mathscr{U}, \mathrm{Z}) \rightarrow \check{\Omega}^{p, 0}(\mathscr{U})
$$

which gives us the quotient complex

$$
\check{\Omega}_{\mathrm{R} / \mathrm{Z}}^{*}(\mathscr{U})=\check{\Omega}^{*}(\mathscr{U}) / \check{C}^{*}(\mathscr{U}, \mathrm{Z}) .
$$

Definition 2.1. 1. An (Hermitian line) $l$-gerbe on $Z$ is an $l$-cocycle in $\check{C}^{l}(\mathscr{U}, \underline{\mathrm{R} / \mathrm{Z}})$ or equivalently a $\theta \in \check{\Omega}_{\mathrm{R} / \mathrm{Z}}^{l, 0}(\mathscr{U})$ with $\delta \theta=0$. 
2. A connection $\omega$ in an $l$-gerbe $\theta$ is a $\omega=\left(\omega_{0}, \ldots, \omega_{l}\right) \in \check{\Omega}^{l}(\mathscr{U})$, where $\omega_{i} \in \check{\Omega}^{i, l-i}(\mathscr{U})$, so that $\omega_{l} \equiv-\theta \bmod \mathbf{Z}$ and the image of $\omega$ is a cocycle in $\check{\Omega}_{\mathrm{R} / \mathrm{Z}}^{*}(\mathscr{U}) / \varepsilon^{*} \Omega^{*}(Z)$.

3. Two $l$-gerbes $\theta$ and $\theta^{\prime}$ with connections $\omega$ and $\omega^{\prime}$ are equivalent if $\omega$ and $\omega^{\prime}$ are cohomologous in $\check{\Omega}_{\mathrm{R} / \mathrm{Z}}^{*}(\mathscr{U})$. The set of equivalence classes $[\theta, \omega]$ is denoted $H_{\mathscr{D}}^{l+1}(Z, Z)$ and is called the $l+1$ 'st smooth Deligne cohomology group.

Remarks 2.2. 1 . Note that $H_{\mathscr{D}}^{l+1}(Z, Z)$ is the cohomology of the sequence

$$
\check{\Omega}_{\mathrm{R} / \mathrm{Z}}^{l-1}(\mathscr{U}) \stackrel{d}{\longrightarrow} \check{\Omega}_{\mathrm{R} / \mathrm{Z}}^{l}(\mathcal{U}) \stackrel{d}{\longrightarrow} \check{\Omega}_{\mathrm{R} / \mathrm{Z}}^{l+1}(\mathscr{U}) / \varepsilon^{*} \Omega^{l+1}(Z) .
$$

2. That the image of $\omega$ is a cocycle in $\check{\Omega}_{\mathrm{R} / \mathrm{Z}}^{*}(\mathscr{U}) / \varepsilon^{*} \Omega^{*}(Z)$ is equivalent to the relations

$$
\delta \omega_{i-1}+(-1)^{i} d \omega_{i}=0, \quad i=1, \ldots, l
$$

and

$$
\delta \omega_{l} \equiv 0 \quad \bmod \mathbf{Z} .
$$

3. Our definition of a gerbe is to some extent an abuse of language, since a gerbe is actually a well-defined geometrical object, so that the set of isomorphism classes of gerbes (with band $\mathrm{R} / \mathrm{Z}$ ) over $Z$ is isomorphic to $H^{2}(Z, R / Z$ ), this corresponds to our case $l=2$. Our viewpoint is analogous to identifying a line bundle with its defining cocycle. If the reader finds this inconvenient, he/she can simply choose to substitute 'gerbe' with 'gerbe data'. See [1] for a thorough exposition of the geometric picture and [10] and the references therein for an alternative approach using 'bundle gerbes'.

Proposition 2.3. 1. We have a commutative diagram

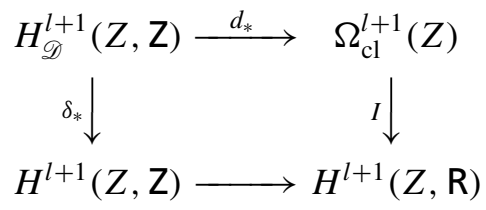

where $\Omega_{\mathrm{cl}}^{l+1}(Z)$ is the set of closed $l+1$-forms with integral periods.

2. There is a short exact sequence

$$
0 \longrightarrow H^{l}(Z, \mathrm{R} / \mathrm{Z}) \longrightarrow H_{\mathscr{D}}^{l+1}(Z, \mathbf{Z}) \stackrel{d_{*}}{\longrightarrow} \Omega_{\mathrm{cl}}^{l+1}(Z) \longrightarrow 0,
$$

Proof. 1. Note that since $\delta d \omega_{0}=d \delta \omega_{0}=d^{2} \omega_{1}=0$ then $F_{\omega}=d \omega_{0}$ is actually a globally defined, closed $l+1$-form. $F_{\omega}$ is called the curvature of $\omega$. 
$d_{*}$ is the map sending $\omega$ to $F_{\omega}$. $\delta_{*}$ is just the connecting homomorphism for the short exact sequence

$$
0 \longrightarrow \mathrm{Z} \longrightarrow \underline{\mathrm{R}} \longrightarrow \underline{\mathrm{R} / \mathrm{Z}} \longrightarrow 0
$$

and $I$ is the de Rham map. Now, commutativity of the diagram follows from the fact that $d \omega_{0}-\delta \theta=d \omega_{0}+\delta \omega_{l}=D \omega$ in $\check{\Omega}^{*}(\mathscr{U})$.

2 . The kernel of $d_{*}$ is the 'gerbes with flat connection'. Since a gerbe with flat connection is actually a cocycle in the full complex $\check{\Omega}_{\mathrm{R} / Z}^{*}(\mathscr{U})$, we see that the kernel is in fact $H^{l}(Z, R / Z)$.

There is also a description of gerbes with connection in terms of the differential characters of Cheeger-Simons [2]. Indeed there is an explicit isomorphism $H_{\mathscr{D}}^{l}(Z, Z) \cong \hat{H}^{l}(Z, Z)$ given in e.g. Dupont-Kamber [5].

\subsection{Simplicial forms}

In [5], there is given a description of gerbes with connection in terms of simplicial forms, which we will briefly recall. (For more details on simplicial forms see [3] or [4, ch. 2]).

Given an open cover $\mathscr{U}=\left\{U_{i}\right\}$ of $Z$ we have the nerve $N \mathscr{U}=\{N \mathscr{U}(p)\}$ of the covering where

$$
N \mathscr{U}(p)=\bigsqcup_{i_{0}, \ldots, i_{p}} U_{i_{0}} \cap \cdots \cap U_{i_{p}} .
$$

We denote $U_{i_{0}} \cap \cdots \cap U_{i_{p}}$ by $U_{i_{0} \ldots i_{p}}$ in the following.

$N \mathscr{U}$ is a simplicial manifold where the face maps

$$
d_{j}: U_{i_{0} \ldots i_{p}} \rightarrow U_{i_{0} \ldots \hat{i_{j} \ldots i_{p}}}
$$

and degeneracy maps

$$
s_{j}: U_{i_{0} \ldots i_{p}} \rightarrow U_{i_{0} \ldots i_{j} i_{j} \ldots i_{p}}
$$

are just inclusions.

DEFINITION 2.5. A simplicial $n$-form $\omega=\left\{\omega^{(p)}\right\}$ on $N \mathscr{U}$ consists of forms $\omega^{(p)} \in \Omega^{n}\left(\Delta^{p} \times N \mathscr{U}(p)\right)$ which satisfy the relations

$$
\left(\varepsilon_{j} \times \mathrm{id}\right)^{*} \omega^{(p)}=\left(\mathrm{id} \times d_{j}\right)^{*} \omega^{(p-1)},
$$

where $\varepsilon_{j}: \Delta^{p-1} \rightarrow \Delta^{p}$ denotes the ordinary $j$ 'th face map. We denote the set of simplicial forms on $N \mathscr{U}$ by $\Omega^{*}(\|N \mathscr{U}\|)$. If the forms also satisfy the relations

$$
\left(\eta_{j} \times \mathrm{id}\right)^{*} \omega^{(p-1)}=\left(\mathrm{id} \times s_{j}\right)^{*} \omega^{(p)},
$$


where $\eta_{j}: \Delta^{p} \rightarrow \Delta^{p-1}$ is the ordinary $j$ 'th degeneracy map, the forms are called normal. The set of normal forms is denoted $\Omega^{*}(|N \mathscr{U}|)$.

Remark 2.6. Our index sets will always be assumed to be ordered and it is then customary to consider only ordered $(p+1)$-tuples, that is for a tuple $\left(i_{0}, \ldots, i_{p}\right)$ we have $i_{0} \leq \cdots \leq i_{p}$. Later when we move on to prism complexes this will in some instances be annoying. Instead we demand that for a permutation $\sigma \in \Sigma(p)$ the normal forms also satisfy the relation

$$
\tilde{\sigma}^{*} \omega=\omega
$$

where $\tilde{\sigma}: \Delta^{p} \times U_{i_{0} \ldots i_{p}} \rightarrow \Delta^{p} \times U_{i_{\sigma(0) \ldots} \ldots i_{\sigma(p)}}$ on the first factor is the simplicial map that permutes the vertices of $\Delta^{p}$ according to $\sigma$ and on the second factor is the identity.

We have a direct sum decomposition

$$
\Omega^{n}(|N \mathscr{U}|)=\bigoplus_{p+q=n} \Omega^{p, q}(|N \mathscr{U}|)
$$

where $\Omega^{p, q}(|N \mathscr{U}|)$ is the set of forms that are of degree $p$ in the barycentric coordinates on the simplex in the product $\Delta^{k} \times N \mathscr{U}(k)$ for $k \geq p$.

There is a chain map

$$
I_{\Delta}: \Omega^{p, q}(|N \mathscr{U}|) \rightarrow \check{\Omega}^{p, q}(\mathscr{U})
$$

given by $I_{\Delta}(\omega)=\int_{\Delta^{p}} \omega^{(p)}$. This map gives an isomorphism in homology. In fact it has a right inverse given on $\Delta^{k} \times N \mathscr{U}(k)$ by

$$
E(\omega)=p ! \sum_{|I|=p} \omega_{I} \wedge d_{I}^{*} \omega
$$

where $I=\left(i_{0}, \ldots, i_{p}\right)$ is a sequence of integers $0 \leq i_{0} \leq \cdots \leq i_{p} \leq k$, $\omega_{I}=\sum_{j=0}^{p}(-1)^{j} t_{i_{j}} d t_{i_{0}} \wedge \cdots \wedge d \hat{t}_{i_{j}} \wedge \cdots \wedge d t_{i_{p}}$ are the elementary forms on $\Delta^{k}$ and $d_{I}: N \mathscr{U}(k) \rightarrow N \mathscr{U}(p)$ is $d_{I}=d_{j_{1}} \cdots d_{j_{l}}$ where $0 \leq j_{l} \leq \cdots \leq j_{1} \leq k$ is the complementary sequence of $I$ (see Dupont $[3, \S 2]$ for details).

The natural map $\sqcup U_{i} \rightarrow Z$ also induces a map

$$
\varepsilon^{*}: \Omega^{*}(Z) \rightarrow \Omega^{*}(|N \mathscr{U}|),
$$

so we get the following commutative diagram of homology isomorphisms:

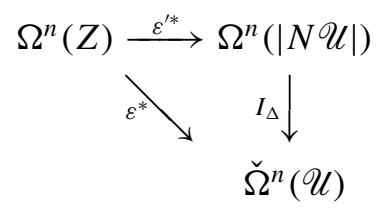


We need a notion of integral simplicial forms in order to imitate the construction in the previous section.

Definition 2.7. A form $\omega \in \Omega^{*}(|N \mathscr{U}|)$ is called discrete if it is locally constant with respect to any local coordinates on the nerve. Furthermore it is called integral if $I_{\Delta}(\omega) \in \check{C}^{*}(\mathcal{U}, \mathrm{Z})$. The chain complex of integral forms is denoted $\Omega_{\mathrm{Z}}^{*}(|N \mathcal{U}|)$

Proposition 2.8. We have the following isomorphisms

1.

$$
H^{n}\left(\Omega_{\mathrm{Z}}^{*}(|N \mathscr{U}|)\right) \cong H^{n}\left(C^{*}(\mathcal{U}, \mathrm{Z})\right)=H^{n}(\mathrm{Z}, \mathrm{Z}) .
$$

2. If we define

$$
\Omega_{\mathrm{R} / \mathrm{Z}}^{*}(|N \mathscr{U}|)=\Omega^{*}(|N \mathscr{U}|) / \Omega_{\mathrm{Z}}^{*}(|N \mathscr{U}|)
$$

then also

$$
H^{*}\left(\Omega_{\mathrm{R} / \mathrm{Z}}^{*}(|N \mathscr{U}|)\right) \cong H^{*}\left(\check{\Omega}_{\mathrm{R} / \mathrm{Z}}^{*}(\mathscr{U})\right) \cong H^{*}(Z, \mathrm{R} / \mathrm{Z}) .
$$

3. $I_{\Delta}$ induces an isomorphism from the cohomology of the sequence

$$
\Omega_{\mathrm{R} / \mathrm{Z}}^{l-1}(|N \mathscr{U}|) \stackrel{d}{\longrightarrow} \Omega_{\mathrm{R} / \mathbf{Z}}^{l}(|N \mathscr{U}|) \stackrel{d}{\longrightarrow} \Omega_{\mathrm{R} / \mathrm{Z}}^{l+1}(|N \mathscr{U}|) / \varepsilon^{*} \Omega^{l+1}(Z)
$$

to $H_{\mathscr{D}}^{l+1}(Z, \mathrm{Z})$.

Proof. 1. The map $I_{\Delta}$ takes integral forms to integral cochains by definition. It induces an isomorphism in cohomology since the map $E$ takes integral cochains to integral forms and the chain homotopies from id to $E \circ I_{\Delta}$ given in $[3, \S 2]$ are easily seen to map integral forms to integral forms.

2. Follows easily from the above.

3. Since the cohomology group of (2.9) fits into a short exact sequence analogous to the one in (2.4) the 5-lemma gives us that $I_{\Delta}$ is an isomorphism.

CoROLlaRy 2.10. Every class in $H_{\mathscr{D}}^{l+1}(Z, Z)$ can be represented by an $l$ gerbe $\theta$ with connection $\omega$, where $\omega=I_{\Delta}(\Lambda)$ for some $\Lambda \in \Omega^{l}(|N \mathscr{U}|)$ and

$$
d \Lambda=\varepsilon^{*} \alpha-\beta, \quad \alpha \in \Omega^{l+1}(Z), \quad \beta \in \Omega_{\mathrm{Z}}^{l+1}(|N \mathscr{U}|) .
$$

\section{Prism complexes}

The notion of a 'prism complex' and 'prismatic' decomposition has occurred (implicitly or explicitly) in many different contexts (see e.g. [11]). We will give further details and references in a forthcoming paper with B. Akyar. 
A prism complex is a generalisation of a simplicial set (or manifold) well suited for fiber bundles. A prism complex $P=\left\{P_{p}\right\}$ is a collection of $(p+1)$ simplicial sets $P_{p}$. That is, for each set of positive integers $\left(q_{0}, \ldots, q_{p}\right)$ we have sets $P_{p, q_{0} \ldots q_{p}}$ with face and degeneracy maps

$$
d_{j}^{i}: P_{p, q_{0} \ldots q_{p}} \rightarrow P_{p, q_{0} \ldots q_{i}-1 \ldots q_{p}}
$$

and

$$
s_{j}^{i}: P_{p, q_{0}, \ldots, q_{p}} \rightarrow P_{p, q_{0} \ldots q_{i}+1 \ldots q_{p}}
$$

for $i=0, \ldots, p, j=0, \ldots, q_{i}$ satisfying the relations

$$
\begin{aligned}
& d_{j}^{i} \circ d_{j^{\prime}}^{i}=d_{j^{\prime}-1}^{i} \circ d_{j}^{i} \quad j<j^{\prime} \\
& s_{j}^{i} \circ s_{j^{\prime}}^{i}=s_{j^{\prime}+1}^{i} \circ s_{j}^{i} \quad j \leq j^{\prime} \\
& d_{j}^{i} \circ s_{j^{\prime}}^{i}= \begin{cases}s_{j^{\prime}-1}^{i} \circ d_{j}^{i} & j<j^{\prime} \\
\mathrm{id} & j=j^{\prime}, j=j^{\prime}+1 \\
s_{j^{\prime}}^{i} \circ d_{j-1}^{i} & j>j^{\prime}+1\end{cases}
\end{aligned}
$$

and so that $s_{j}^{i}$ and $d_{j}^{i}$ commute with $s_{j^{\prime}}^{i^{\prime}}$ and $d_{j^{\prime}}^{i^{\prime}}$ for $i \neq i^{\prime}$.

Furthermore we want another set of simplicial (i.e. commuting with the $d_{j}^{i}$, s and $s_{j}^{i}$,s) face maps $d_{i}: P_{p, q_{0} \ldots q_{p}} \rightarrow P_{p-1, q_{0} \ldots \hat{q}_{i} \ldots q_{p}}$ and degeneracy maps $s_{i}$ : $P_{p, q_{0} \ldots q_{p}} \rightarrow P_{p+1, q_{0}, \ldots q_{i} q_{i} \ldots q_{p}}$ so that $\left(P_{p}, d_{i}, s_{i}\right)$ becomes an ordinary simplicial set. Note that in some applications the last set of degeneracy maps does not exist naturally so in these cases $\left(P_{p}, d_{i}\right)$ is only a $\Delta$-set. As with ordinary simplicial sets we can for each $p$ form the geometric and fat realisations $\left|P_{p}\right|$ and $\left\|P_{p}\right\|$, that is, the quotients of

$$
\bigsqcup_{q_{0} \ldots q_{p}} \Delta^{q_{0}} \times \cdots \times \Delta^{q_{p}} \times P_{p, q_{0} \ldots q_{p}}
$$

where we divide out by the equivalence relations generated by the face and degeneracy maps

$$
\varepsilon_{j}^{i}: \Delta^{q_{0} \ldots q_{i} \ldots q_{p}} \rightarrow \Delta^{q_{0} \ldots q_{i}+1 \ldots q_{p}}
$$

and (in case of the geometric realisation)

$$
\eta_{j}^{i}: \Delta^{q_{0} \ldots q_{i} \ldots q_{p}} \rightarrow \Delta^{q_{0} \ldots q_{i}-1 \ldots q_{p}}
$$

(where $\Delta^{q_{0} \ldots q_{p}}$ is short hand notation for the prism $\Delta^{q_{0}} \times \cdots \times \Delta^{q_{p}}$ ).

The face and degeneracy maps $d_{i}$ and $s_{i}$ now induce a structure of a simplicial set on $\left|P_{p}\right|\left(\left\|P_{p}\right\|\right)$ by acting as the projection and the diagonal on 
$\Delta^{q_{0}} \times \cdots \times \Delta^{q_{p}}$ respectively. That is let $\pi_{i}: \Delta^{q_{0} \ldots q_{p}} \rightarrow \Delta^{q_{0} \ldots \hat{q}_{i} \ldots q_{p}}$ be the projection that deletes the $i$ 'th coordinate and let $\Delta_{i}: \Delta^{q_{0} \ldots q_{p}} \rightarrow \Delta^{q_{0} \ldots q_{i} q_{i} \ldots q_{p}}$ be the diagonal map that repeats the $i$ 'th factor. Then we can form the geometric realisation

$$
|P .|=\bigsqcup_{p \geq 0} \Delta^{p} \times\left|P_{p}\right| / \sim
$$

where the equivalence relation is generated by

$$
\left(\varepsilon_{i} t, s, x\right) \sim\left(t, \pi_{i} s, d_{i} x\right), \quad t \in \Delta^{p-1}, s \in \Delta^{q_{0} \ldots q_{p}}, x \in P_{p, q_{0} \ldots q_{p}}
$$

and

$$
\left(\eta_{i} t, s, x\right) \sim\left(t, \Delta_{i} s, s_{i} x\right), \quad t \in \Delta^{p+1}, s \in \Delta^{q_{0} \ldots q_{p}}, x \in P_{p, q_{0} \ldots q_{p}}
$$

Example 3.1. Given a smooth fiber bundle $\pi: Y \rightarrow Z$ with $\operatorname{dim} Y=$ $m+n, \operatorname{dim} Z=m$ and compact fibers, possibly with boundary, a theorem of Johnson [9] gives us smooth triangulations $K$ and $L$ of $Y$ and $Z$ respectively and a simplicial map $\pi^{\prime}: K \rightarrow L$ so that the following diagram commutes

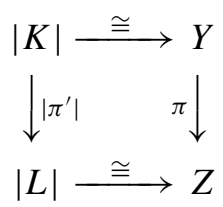

Here the horizontal maps are homeomorphisms which are smooth on each simplex. Furthermore given such a triangulation of $\partial Y \rightarrow Z$ we can also extend it to a triangulation of $Y \rightarrow Z$.

Now the geometric idea is that if $z \in Z$ lies in the interior of a $p$-simplex of $L$ then the fiber over $z$ is in a canonical way decomposed into $(p+1)$-fold prisms of the form $\Delta^{q_{0} \ldots q_{p}}$ as above. Formally we define the prismatic complex $P S(K / L)$ by letting $P S_{p}(K / L)_{q_{0} \ldots q_{p}} \subseteq S_{p+q_{0}+\cdots+q_{p}}(K) \times S_{p}(L)$ be the subset of pairs of simplices $(\tau, \eta)$ so that $q_{i}+1$ of the vertices in $\tau$ lie over the $i$ 'th vertex in $\eta$. Then we have face and degeneracy operators defined in the obvious way. In particular let $P C_{p}(K / L)_{q_{0} \ldots q_{p}}$ be the free abelian group generated by $P S_{p}(K / L)_{q_{0} \ldots q_{p}}$ then this gives us boundary maps in the fiber direction of the associated chain complex

$$
\partial_{F}^{i}: P C_{p}(K / L)_{q_{0} \ldots q_{p}} \rightarrow P C_{p}(K / L)_{q_{0} \ldots q_{i}-1 \ldots q_{p}}
$$

defined by $\partial_{F}^{i}=\sum(-1)^{j} d_{j}^{i}$, ( $\partial_{F}^{i}=0$ for $\left.q_{i}=0\right)$, and also a total boundary map along the fiber

$$
\partial_{F}=\partial_{F}^{0}+(-1)^{q_{0}+1} \partial_{F}^{1}+\cdots+(-1)^{q_{0}+\cdots+q_{p-1}+p} \partial_{F}^{p} .
$$


Also there is a horizontal boundary map

$$
\partial_{H}=\partial_{0}+(-1)^{q_{0}+1} \partial_{1}+\cdots+(-1)^{q_{0}+\cdots+q_{p-1}+p} \partial_{p},
$$

where

$$
\partial_{i}= \begin{cases}0 & \text { if } q_{i}>0 \\ d_{i} & \text { if } q_{i}=0\end{cases}
$$

so that $\partial=\partial_{F}+\partial_{H}$ is a boundary map in the total complex $P C_{*}(K / L)$ of $P C_{*}(K / L)_{* \ldots *}$. This is actually the cellular chain complex for the geometric realisation and hence calculates the homology of $Y$.

There is a natural 'prismatic triangulation' homeomorphism

$$
\ell:|P S(K / L)| \stackrel{\cong}{\longrightarrow}|K|
$$

induced by

$$
\ell\left(t, s^{0}, \ldots, s^{p},(\tau, \eta)\right)=\left(t_{0} s^{0}, \ldots, t_{p} s^{p}, \tau\right)
$$

for $(t, s, \tau) \in \Delta^{p} \times \Delta^{q_{0} \ldots q_{p}} \times P S_{p}(K / L)_{q_{0} \ldots q_{p}}$. Note that if $\stackrel{\circ}{\sigma}$ is an open $p$-simplex in $L$ then $\ell$ provides a natural trivialisation of $|K|$ over $\stackrel{\circ}{\sigma}$

$$
\stackrel{\circ}{\sigma} \times\left|P S_{p}(K / \sigma)\right| \stackrel{\cong}{\longrightarrow}|K|_{\mid \sigma}
$$

Example 3.2. Another example in the category of manifolds, comes from the nerve of compatible open coverings of the total space and the base space. That is, given a covering $\mathscr{U}=\left\{U_{i}\right\}$ of $Z$ we have a covering $\mathscr{W}=\left\{W_{i}=\right.$ $\left.\pi^{-1}\left(U_{i}\right)\right\}$ of $Y$, and for each $i, \mathscr{V}^{i}$ is an open cover of $W_{i}$. This gives a covering $\mathscr{V}=\cup \mathscr{V}^{i}$ of $Y$ (with lexicographically ordered index set). Then we put

$$
P_{p} N(\mathscr{V} / \mathcal{U})_{q_{0} \ldots q_{p}}=\bigsqcup V_{j_{0}^{0}}^{i_{0}} \cap \cdots \cap V_{j_{q_{0}}^{0}}^{i_{0}} \cap \cdots \cap V_{j_{q_{p}}^{p}}^{i_{p}}
$$

with $V_{j}^{i} \in \mathscr{V}^{i}$, and face and degeneracy maps are inclusions similar to the simplicial case in section 2.1. In the following, we will denote $V_{j_{0}^{0}}^{i_{0}} \cap \cdots \cap V_{j_{q p}^{p}}^{i_{p}}$ by $V_{j_{0}^{0} \ldots j_{p}^{p}}$.

A useful special case of this situation occurs in the context of example 3.1 above with the coverings consisting of the (open) stars of the triangulations of $K$ and $L$. More precisely $\mathscr{U}=\left\{U_{i}=\operatorname{st}\left(a_{i}\right)\right\}$ where $a_{i} \in L^{0}$ is a 0 -simplex in $L$ and $\mathscr{V}^{i}=\left\{V_{j}^{i}=\operatorname{st}\left(b_{j}^{i}\right)\right\}$ where $b_{j}^{i} \in \pi^{-1}\left(a_{i}\right) \cap K^{0}$. 


\subsection{Prismatic forms}

As a straightforward generalisation of simplicial forms, we introduce the complex of (normal) prismatic forms on the prism complex in the above example 3.2.

Definition 3.3. A prismatic $n$-form is a collection $\omega=\left\{\omega_{q_{0} \ldots q_{p}}\right\}$ of forms $\omega_{q_{0} \ldots q_{p}} \in \Omega^{n}\left(\Delta^{p} \times \Delta^{q_{0} \ldots q_{p}} \times P_{p} N(\mathscr{V} / \mathscr{U})_{q_{0} \ldots q_{p}}\right)$ satisfying the relations

$$
\left(\mathrm{id} \times \varepsilon_{j}^{i} \times \mathrm{id}\right)^{*} \omega_{q_{0} \ldots q_{p}}=\left(\mathrm{id} \times \mathrm{id} \times d_{j}^{i}\right)^{*} \omega_{q_{0} \ldots q_{i}-1 \ldots q_{p}}
$$

and

$$
\left(\varepsilon_{i} \times \mathrm{id} \times \mathrm{id}\right)^{*} \omega_{q_{0} \ldots q_{p}}=\left(\mathrm{id} \times \pi_{i} \times d_{i}\right)^{*} \omega_{q_{0} \ldots \hat{q}_{i} \ldots q_{p}} .
$$

A form is called normal if it also satisfies the relations

$$
\left(\mathrm{id} \times \eta_{j}^{i} \times \mathrm{id}\right)^{*} \omega_{q_{0} \ldots q_{i}-1 \ldots q_{p}}=\left(\mathrm{id} \times \mathrm{id} \times s_{j}^{i}\right)^{*} \omega_{q_{0} \ldots q_{p}}
$$

and

$$
\left(\eta_{i} \times \mathrm{id} \times \mathrm{id}\right)^{*} \omega_{q_{0} \ldots q_{p}}=\left(\mathrm{id} \times \Delta_{i} \times s_{i}\right)^{*} \omega_{q_{0} \ldots q_{i} q_{i} \ldots q_{p}} .
$$

The complex of normal prismatic forms is denoted by

$$
\Omega^{*}(|P N \mathscr{V} / \mathscr{U}|) .
$$

As in the simplicial case we have a direct sum decomposition of this complex

$$
\begin{aligned}
\Omega^{n}(|P N \mathscr{V}| \mathscr{U} \mid) & =\bigoplus_{p+q+r=n} \Omega^{p, q, r}(|P N \mathscr{V} / \mathscr{U}|) \\
& =\bigoplus_{p+q_{0}+\cdots+q_{p}+r=n} \Omega^{p, q_{0}, \ldots, q_{p}, r}(|P N \mathscr{V}| \mathscr{U} \mid),
\end{aligned}
$$

where $\Omega^{p, q_{0}, \ldots, q_{p}, r}(|P N \mathscr{V} / \mathscr{U}|)$ is the set of forms of degree $p$ in the barycentric coordinates of the first simplex, of degree $q_{0}$ in the second and so on and finally of degree $r$ in some local coordinates on the nerve of the covering. This makes $\Omega^{*}(|P N \mathscr{V} / \mathscr{U}|)$ into a triple-complex. There is also a corresponding Čech-de Rham triple-complex

$$
\check{\Omega}^{p, q, r}(\mathscr{V} / \mathscr{U})=\bigoplus_{q_{0}+\ldots+q_{p}=q} \Omega^{r}\left(P_{p} N(\mathscr{V} / \mathscr{U})_{q_{0}, \ldots, q_{p}}\right)
$$

with differentials

$$
\begin{aligned}
\partial^{\prime}: \check{\Omega}^{p, q, r}(\mathscr{V} / \mathscr{U}) & \rightarrow \check{\Omega}^{p+1, q, r}(\mathscr{V} / \mathscr{U}) \\
\partial^{\prime \prime}: \check{\Omega}^{p, q, r}(\mathscr{V} / \mathscr{U}) & \rightarrow \check{\Omega}^{p, q+1, r}(\mathscr{V} / \mathscr{U}) \\
\partial^{\prime \prime \prime}: \check{\Omega}^{p, q, r}(\mathscr{V} / \mathscr{U}) & \rightarrow \check{\Omega}^{p, q, r+1}(\mathscr{V} / \mathscr{U})
\end{aligned}
$$


Here $\partial^{\prime}=\sum(-1)^{i} \partial_{i}^{\prime}$ where

$$
\partial_{i}^{\prime} \alpha_{\mid j_{0}^{0} \ldots j_{q_{p+1}}^{p+1}}= \begin{cases}0 & \text { if } q_{i}>0 \\ \alpha_{\mid j_{0}^{0} \ldots \hat{j}_{0}^{i} \ldots j_{q_{p+1}}^{p+1}} & \text { if } q_{i}=0\end{cases}
$$

$\partial^{\prime \prime}$ and $\partial^{\prime \prime \prime}$ are usual Čech and de Rham differentials.

As in the simplicial case we have

Proposition 3.4. The map

$$
I_{\Delta}: \Omega^{p, q, r}(|P N \mathscr{V} / \mathscr{U}|) \rightarrow \check{\Omega}^{p, q, r}(\mathscr{V} / \mathscr{U})
$$

given by

$$
I_{\Delta}(\omega)=\int_{\Delta^{p} \times \Delta^{q_{0} \ldots q_{p}}} \omega_{q_{0} \ldots q_{p}}, \quad \text { for } \quad \omega \in \Omega^{p, q_{0}, \ldots, q_{p}, r}(|P N \mathscr{V} / \mathscr{U}|)
$$

induces an isomorphism in cohomology. The right inverse is given on $\Delta^{k_{0} \ldots k_{p}} \times$ $P_{p} N(\mathscr{V} / \mathscr{U})_{k_{0} \ldots k_{p}}$ by

$$
E(\omega)=p ! q_{0} ! \cdots q_{p} ! \sum_{|J|=p} \sum_{\left|J_{0}\right|=q_{0}} \cdots \sum_{\left|J_{p}\right|=q_{p}} \omega_{J} \wedge \omega_{J_{0}} \wedge \cdots \wedge \omega_{J_{p}} \wedge d_{J_{0} \cdots J_{p}}^{*} \omega .
$$

The $\omega_{J_{j}}$ 's are the elementary forms on $\Delta^{q_{j}}$ and $d_{J_{0} \cdots J_{p}}$ are face maps as in the simplicial case.

Proof. The proof is the same as in the simplicial case (see e.g. [3]).

Proposition 3.5. The inclusion $\varepsilon: \sqcup V_{j}^{i} \rightarrow W_{i}$ induces the maps $\varepsilon_{1}^{*}$ and $\varepsilon_{2}^{*}$ in the following commutative diagram

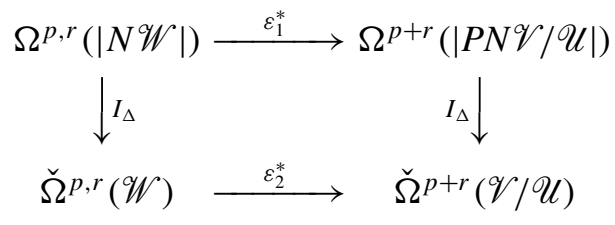

They both induce isomorphisms in cohomology.

Proof. We first notice that both $\Omega^{p, 0, r}(|P N \mathscr{V} / \mathscr{U}|) \cong \Omega^{p, r}(|N \mathscr{V}|)$ and $\check{\Omega}^{p, 0, r}(\mathscr{V} / \mathscr{U}) \cong \check{\Omega}^{p, r}(\mathscr{V})$ as double complexes, so in the following diagram

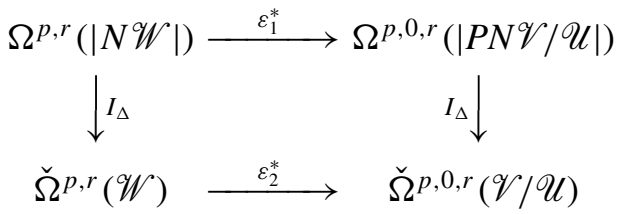


the $\varepsilon_{i}$ 's are just refinement maps and thus induce isomorphisms in cohomology.

Now let us see that for fixed $p$ and $r$ the complex $\check{\Omega}^{p, q, r}(\mathscr{V} / \mathscr{U})$ is exact, this will imply that

$$
\check{\Omega}^{p, 0, r}(\mathscr{V} / \mathscr{U}) \rightarrow \check{\Omega}^{p+r}(\mathscr{V} / \mathscr{U})
$$

is a cohomology isomorphism.

We first construct homomorphisms

$$
s^{i}: \check{\Omega}^{p, q_{0}, \ldots, q_{p}, r}(\mathscr{V} / \mathscr{U}) \rightarrow \check{\Omega}^{p, q_{0}, \ldots, q_{i}-1, \ldots q_{p}}(\mathscr{V} / \mathcal{U}) .
$$

We choose partitions of unity on $W_{i}$ subordinate $\mathscr{V}^{i}=\left\{V_{j}^{i}\right\}_{j \in J_{i}}$ for each $i \in I$ and set

$$
s^{i}(\omega)_{j_{0}^{0} \ldots j_{q_{i}-1}^{i} j_{0}^{i+1} \ldots j_{q_{p}}^{p}}=(-1)^{q_{0}+\cdots+q_{i}} \sum_{j \in J_{i}} \phi_{j}^{i} \omega_{j_{0}^{0} \ldots j_{q_{i}-1}^{i} j j_{0}^{i+1} \ldots j_{q_{p}}^{p}}
$$

Let $\tilde{\delta}^{i}=(-1)^{q_{0}+\cdots+q_{i-1}} \delta^{i}$, then we have

$$
s^{i} \tilde{\delta}^{j}+\tilde{\delta}^{j} s^{i}=0, \quad i \neq j
$$

and

$$
s^{i} \tilde{\delta}^{i}+\tilde{\delta}^{i} s^{i}=\mathrm{id} .
$$

This gives

$$
s^{i} \delta+\delta s^{i}=\mathrm{id}
$$

for each $i$. So for fixed $p$ and $r$ the chain complex $\check{\Omega}^{q, r}(\mathscr{V} / \mathscr{U})$ is exact.

COROLlary 3.6. We have a quasi-isomorphism

$$
\varepsilon^{*}: \Omega^{*}(|N \mathscr{W}|) \rightarrow \Omega^{*}(|P N \mathscr{V} / \mathscr{U}|)
$$

induced by the inclusion $\sqcup V_{j}^{i} \rightarrow W_{i}$.

REMARK 3.7. The above result could have been obtained in a different manner. In the next section, we will construct a right inverse $\phi$ to $\varepsilon^{\prime}$. We could then have constructed a homotopy $\phi \circ \varepsilon^{\prime} \sim$ id which would give us a chain homotopy directly on $\Omega^{*}(|P N \mathscr{V} / \mathscr{U}|)$.

\section{Integration}

For a fiber bundle with compact, oriented $n$-dimensional fibers, we want to define an integration map $\int: \Omega^{k+n}(|N \mathscr{V}|) \rightarrow \Omega^{k}(|N \mathscr{U}|)$ for coverings $\mathscr{U}$ and $\mathscr{V}$ coming from triangulations as in example 3.2. To do so, we define a map $|N \mathscr{W}| \rightarrow|N \mathscr{V}|$, and then our integration is given by pulling back forms by 
this map and then integrating along the fiber in $|N \mathscr{W}| \rightarrow|N \mathscr{U}|$. We define the map in two steps. First we have, similar to the 'prismatic triangulation' map in example 3.1, a map $\ell:|P N \mathscr{V} / \mathscr{U}| \rightarrow|N \mathscr{V}|$ defined on

$$
\ell: \Delta^{p} \times \Delta^{q_{0} \ldots q_{p}} \times V_{j_{0}^{0} \ldots j_{q_{p}}^{p}} \rightarrow \Delta^{p+q_{0}+\cdots+q_{p}} \times V_{j_{0}^{0} \ldots j_{q_{p}}^{p}}
$$

by

$$
\ell\left(t, s^{0}, \ldots, s^{p}, x\right)=\left(t_{0} s^{0}, \ldots, t_{p} s^{p}, x\right)
$$

Now recall that each $W_{i}$ is covered by $\mathscr{V}^{i}=\left\{\operatorname{st}\left(b_{j}^{i}\right)\right\}_{j \in J_{i}}$. Choose partitions of unity $\left\{\phi_{j}^{i}\right\}$ for $W_{i}$ subordinate $\mathscr{V}^{i}$ for each $i$. We are now ready to define

$$
\tilde{\phi}:|N \mathscr{W}| \rightarrow|P N \mathscr{V} / \mathscr{U}|
$$

on $\Delta^{p} \times W_{i_{0} \ldots i_{p}}$. Take $x \in W_{i_{0} \ldots i_{p}}$. For each $i=i_{0}, \ldots, i_{p}$ there is a minimal set $\left\{j_{0}^{i}, \ldots j_{q_{i}}^{i}\right\} \in J_{i}$ so that

$$
\sum_{r=0}^{q_{i}} \phi_{j_{r}^{i}}^{i}(x)=1
$$

We then map

$$
(t, x) \in \Delta^{p} \times W_{i_{0} \ldots i_{p}}
$$

to

$$
\left(t, \phi_{j_{0}^{0}}^{i_{0}}(x), \ldots, \phi_{j_{q_{0}}^{0}}^{i_{0}}(x), \ldots, \phi_{j_{q p}^{p}}^{i_{p}^{p}}(x), x\right) \in \Delta^{p} \times \Delta^{q_{0} \ldots q_{p}} \times V_{j_{0}^{0} \cdots j_{q_{p}}^{p}}
$$

REMARK 4.1. For later use we note that since the covering $\mathscr{V}$ comes from a triangulation it has covering dimension $n+m$ so we have ensured that $q=\sum q_{i} \leq n$ for non-degenerate prisms.

Now for $\omega \in \Omega^{n+k}(|N \mathscr{V}|)$ define $\int_{[Y / Z]} \omega \in \Omega^{k}(|N \mathscr{U}|)$ by

$$
\left(\int_{[Y / Z]} \omega\right)_{\mid \Delta^{p} \times U_{i_{0} \ldots i_{p}}}=\int_{\Delta^{p} \times W_{i_{0} \ldots i_{p} / \Delta^{p} \times U_{i_{0} \ldots i_{p}}}} \tilde{\phi}^{*} \ell^{*} \omega,
$$

where the right hand side denotes usual integration along the fibers.

THEOREM 4.2. Given triangulations and partitions of unity as above, the following holds.

1. Let $\omega \in \Omega^{*+n}\left(\left|N^{\mathscr{V}}\right|\right)$ be a normal simplicial form, then $\int_{[Y / Z]} \omega$ is a well-defined normal simplicial form. 
2. For $\omega \in \Omega^{*+n-1}(|N \mathscr{V}|)$ we have

$$
\int_{[Y / Z]} d \omega=\int_{[\partial Y / Z]} \omega+(-1)^{n} d \int_{[Y / Z]} \omega .
$$

Proof. 1. It is clear that $\int_{[Y / Z]} \omega$ is a well-defined simplicial form i.e. is compatible with respect to the degeneracy operators. Let us see that it is normal, that is

$$
\left(\eta_{j} \times \mathrm{id}\right)^{*}\left(\int_{[Y / Z]} \omega\right)^{(p)}=\left(\mathrm{id} \times s_{j}\right)^{*}\left(\int_{[Y / Z]} \omega\right)^{(p+1)}
$$

We first notice that

$$
\begin{aligned}
\left(\eta_{j} \times \mathrm{id}\right)^{*}\left(\int_{[Y / Z]} \omega\right)_{\mid \Delta^{p} \times U_{i_{0} \ldots i_{p}}} & =\left(\eta_{j} \times \mathrm{id}\right)^{*} \int_{\Delta^{p} \times W_{i_{0} \ldots i_{p}} / \Delta^{p} \times U_{i_{0} \ldots i_{p}}} \tilde{\phi}^{*} \ell^{*} \omega \\
& =\int_{\Delta^{p+1} \times W_{i_{0} \ldots i_{p}} / \Delta^{p+1} \times U_{i_{0} \ldots i_{p}}}\left(\eta_{j} \times \mathrm{id}\right)^{*} \tilde{\phi}^{*} \ell^{*} \omega \\
& =\int_{\Delta^{p+1} \times W_{i_{0} \ldots i_{p}} / \Delta^{p+1} \times U_{i_{0} \ldots i_{p}}} \tilde{\phi}^{*}\left(\eta_{j} \times \mathrm{id}\right)^{*} \ell^{*} \omega \\
& =\int_{\Delta^{p+1} \times W_{i_{0} \ldots i_{p}} / \Delta^{p+1} \times U_{i_{0} \ldots i_{p}}} \tilde{\phi}^{*}\left(\ell \circ\left(\eta_{j} \times \mathrm{id}\right)\right)^{*} \omega
\end{aligned}
$$

and at the same time

$$
\begin{aligned}
\left(\mathrm{id} \times s_{j}\right)^{*} & \left(\int_{[Y / Z]} \omega\right)_{\mid \Delta^{p+1} \times U_{i_{0} \ldots i_{j} i_{j} \ldots i_{p}}} \tilde{\phi}^{*} \ell^{*} \omega \\
& =\left(\mathrm{id} \times s_{j}\right)^{*} \int_{\Delta^{p+1} \times W_{i_{0} \ldots i_{j} i_{j} \ldots i_{p}} / \Delta^{p+1} \times U_{i_{0} \ldots i_{j} i_{j} \ldots i_{p}}}\left(\mathrm{id} \times s_{j}\right)^{*} \tilde{\phi}^{*} \ell^{*} \omega \\
& =\int_{\Delta^{p+1} \times W_{i_{0} \ldots i_{j} \ldots i_{p}} / \Delta^{p+1} \times U_{i_{0} \ldots i_{j} \ldots i_{p}}} \tilde{\phi}^{*}\left(\mathrm{id} \times s_{j}\right)^{*} \ell^{*} \omega \\
& =\int_{\Delta^{p+1} \times W_{i_{0} \ldots i_{p}} / \Delta^{p+1} \times U_{i_{0} \ldots i_{p}}} \tilde{\phi}^{*}\left(\ell \circ\left(\mathrm{id} \times s_{j}\right)\right)^{*} \omega . \\
& =\int_{\Delta^{p+1} \times W_{i_{0} \ldots i_{p}} / \Delta^{p+1} \times U_{i_{0} \ldots i_{p}}}
\end{aligned}
$$

Hence we only need to show that $\left(\ell \circ\left(\mathrm{id} \times s_{j}\right)\right)^{*} \omega=\left(\ell \circ\left(\eta_{j} \times \text { id }\right)\right)^{*} \omega$. This 
can be seen from the following commutative diagram

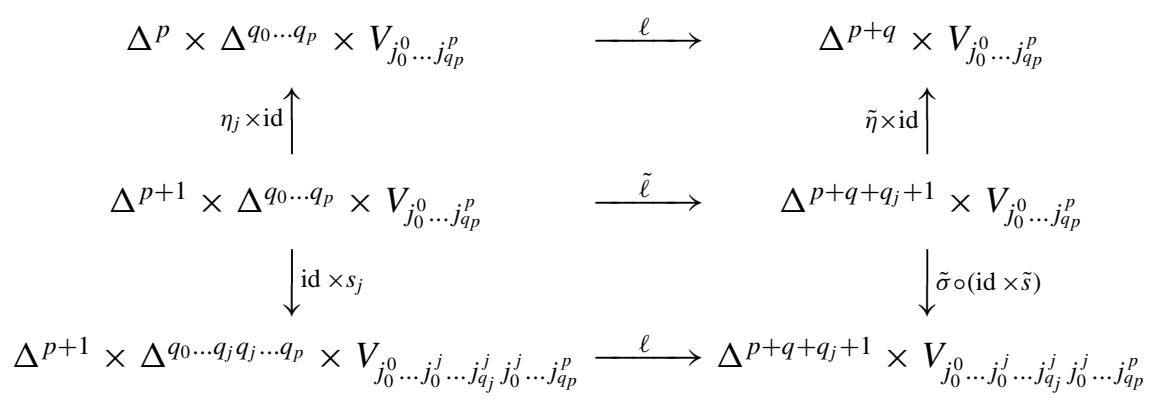

where $q=\sum q_{i}, \tilde{\ell}$ is given by

$\tilde{\ell}\left(t, s^{0}, \ldots, s^{p}, x\right)=\left(t_{0} s^{0}, \ldots, t_{j} s_{0}^{j}, t_{j+1} s_{0}^{j}, t_{j} s_{1}^{j}, \ldots, t_{j+1} s_{q_{j}}^{j}, \ldots, t_{p+1} s^{p}, x\right)$,

$\tilde{\eta}$ (and similarly for $\tilde{s}$ ) is given by

$$
\tilde{\eta}=\eta_{q_{0}+\cdots+q_{j-1}+j} \circ \eta_{q_{0}+\cdots+q_{j-1}+j+2} \circ \cdots \circ \eta_{q_{0}+\cdots+q_{j-1}+j+2 q_{j}}
$$

and finally $\tilde{\sigma}$ is the map that permutes the vertices in the simplex as in remark 2.6 , so that by assumption $\tilde{\sigma}^{*} \omega=\omega$.

2. Follows from the analogous formula for usual fiber integration.

There is a map $\varepsilon^{\prime}:|N \mathscr{V}| \rightarrow|N \mathscr{W}|$ induced by the inclusions $\sqcup V_{j}^{i} \rightarrow W_{i}$ given on $\Delta^{p+q_{0}+\cdots+q_{p}} \times V_{j_{0}^{0} \ldots j_{q_{p}}^{p}}$ by

$$
\varepsilon^{\prime}\left(t_{0}^{0}, \ldots, t_{q_{0}}^{0}, \ldots, t_{q_{p}}^{p}, x\right)=\left(\sum_{j} t_{j}^{0}, \ldots, \sum_{j} t_{j}^{p}, x\right) \in \Delta^{p} \times W_{i_{0} \ldots i_{p}} .
$$

Since $\varepsilon^{\prime}$ is left inverse to $\ell \circ \tilde{\phi}$ the following lemma follows easily from the construction of the integral

Lemma 4.3. The following diagrams commute

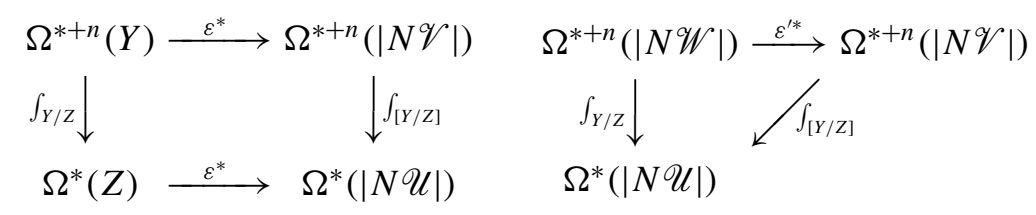

that is the integration of simplicial forms is compatible with the usual fiber integration. 


\section{A combinatorial formula}

As in the case of a product bundle (cf. Gomi-Terashima [7]) there is also a combinatorial formula for the integration map in the case of a general fiber bundle. However, the resulting forms are only piecewise smooth. We start by introducing a new complex consisting of these forms.

\subsection{The triangulated nerve}

Given a triangulation $L$ of a smooth $m$-dimensional manifold $Z$ we have as mentioned earlier an open cover $\mathscr{U}$ given by the stars st $(a)$ where $a$ is a point in $L^{0}$.

For every simplex $\sigma \in L$, the closed star

$$
\overline{\operatorname{st}(\sigma)}=\bigcup_{\tau \in L^{m}, \sigma \subseteq \tau}|\tau|
$$

inherits a natural triangulation $L_{\sigma}$ from $L$. This gives a realisation $\left|L_{\sigma}\right| \cong$ $\overline{\operatorname{st}(\sigma)}$.

Definition 5.1. The triangulated nerve $N L$ is the simplicial complex with $p$-simplices given by

$$
N_{p} L=\bigsqcup_{\sigma \in L^{p}}\left|L_{\sigma}\right|
$$

and for $\sigma=\left[a_{0}, \ldots, a_{p}\right]$ the face and degeneracy operators

$$
d_{j}:\left|L_{a_{0} \ldots a_{p}}\right| \rightarrow\left|L_{a_{0} \ldots \hat{a}_{j} \ldots a_{p}}\right| \quad \text { and } \quad s_{j}:\left|L_{a_{0} \ldots a_{p}}\right| \rightarrow\left|L_{a_{0} \ldots a_{j} a_{j} \ldots a_{p}}\right|
$$

are given by inclusions.

Our construction will give simplicial forms on $|N L|$.

Recall that a form $\omega$ on a simplicial complex is a collection of forms $\omega=$ $\left\{\omega^{(p)}\right\}$ with $\omega^{(p)} \in \Omega^{*}\left(\Delta^{p} \times N_{p} L\right)$ satisfying the relation $\left(\varepsilon^{j} \times \mathrm{id}\right)^{*} \omega^{(p)}=$ (id $\left.\times d_{j}\right)^{*} \omega^{(p-1)}$. But the $L_{\sigma}$ 's, $\sigma \in L^{p}$, are simplicial sets too, so our forms $\omega^{(p)}$ actually live on

$$
\sqcup_{\sigma \in L^{p}} \sqcup_{i} \Delta^{p} \times \Delta^{i} \times L_{\sigma}^{(i)},
$$

where $L_{\sigma}^{(i)}$ is the discrete set of $i$-simplices in $L_{\sigma}$.

Now much of what has been done in the previous sections carry over. We can define integral forms $\Omega_{\mathrm{z}}^{*}(|N L|) \subseteq \Omega^{*}(|N L|)$ exactly as before and given triangulations of a fiber bundle as in example 3.1 we also get triangulated nerves both of the base and the total space. We can also associate a prism complex to this situation in exactly the same way as in example 3.2. There is obviously also a map $\ell:|P N K / L| \rightarrow|N K|$ as before. 
Now let us show that with regard to cohomology it does not matter whether we use ordinary simplicial forms or simplicial forms on the triangulated nerves.

We introduce the simplicial manifold (with corners) $N \overline{\mathscr{U}}$ with

$$
N_{p} \overline{\mathscr{U}}=\bigsqcup_{i_{0}, \ldots, i_{p}} \overline{U_{i_{0} \ldots i_{p}}} .
$$

Since the cohomology of $\Omega^{*}(|N \mathscr{U}|)$ does not depend on the open cover and since forms on a closed subset are restrictions of forms on a larger open subset, we get that the restriction $\Omega^{*}(|N \overline{\mathscr{U}}|) \rightarrow \Omega^{*}(|N \mathscr{U}|)$ induces an isomorphism in cohomology.

Proposition 5.2. The map

$$
\iota: \Omega^{*}(|N \overline{\mathscr{U}}|) \rightarrow \Omega^{*}(|N L|)
$$

induced by the homeomorphisms

$$
\left|L_{a_{0} \ldots a_{p}}\right| \cong \overline{\operatorname{st}\left(\left[a_{0}, \ldots, a_{p}\right]\right)}
$$

is an isomorphism in cohomology.

PROOF. The result follows readily from the following commutative diagram

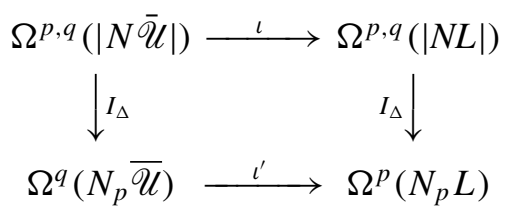

since both vertical maps are isomorphisms in cohomology by the simplicial de Rham theorem. In fact, the de Rham theorem also implies that the lower horizontal map induces an isomorphism in cohomology, since the map $\Omega^{q}(\overline{\operatorname{st}(\sigma)}) \rightarrow \Omega^{q}\left(\left|L_{\sigma}\right|\right)$ is a cohomology isomorphism for all $\sigma \in L$.

Now let us show that we can also represent a class in Deligne cohomology by a simplicial form on a triangulated nerve.

First, let $\mathscr{U}=\left\{U_{i}\right\}_{i \in I}$ be a covering of $Z$ and let $L$ be a triangulation, so that every closed star of $L$ lies inside an open set of $\mathcal{U}$. That is we have a map $\alpha: L^{0} \rightarrow I$ so that $\overline{\operatorname{st}(a)} \subseteq U_{\alpha(a)}$. This gives a chain map

$$
T: \Omega^{*}(|N \mathscr{U}|) \rightarrow \Omega^{*}(|N L|)
$$

Proposition 5.3. The map $T$ induces an isomorphism both in ordinary cohomology and between the cohomology of (2.9) and

$$
\Omega_{\mathrm{R} / \mathrm{Z}}^{l-1}(|N L|) \stackrel{d}{\longrightarrow} \Omega_{\mathrm{R} / \mathrm{Z}}^{l}(|N L|) \stackrel{d}{\longrightarrow} \Omega^{l+1}(|N L|) / \varepsilon^{*} \Omega^{l+1}(Z)
$$


Proof. Follows from the last proposition since $T$ is the composition of a refinement map and $\iota$.

Hence smooth Deligne cohomology is represented by the cohomology of the sequence (5.4).

\subsection{The integration map}

We want to define an integration map

$$
\int_{K / L}: \Omega^{*+n}(|N K|) \rightarrow \Omega^{*}(|N L|)
$$

First, for a simplex $\sigma=\left[a_{0}, \ldots, a_{p}\right] \in L$ we define a map

$$
\mathrm{AW}: P C_{k, m}\left(K / L_{\sigma}\right) \rightarrow \bigoplus_{k_{1}+k_{2}=k} P C_{k_{1}, p}(K / \sigma) \otimes P C_{k_{2}, m}\left(K / L_{\sigma}\right)
$$

Let $(\tau, \eta) \in P C_{k, m}\left(K / L_{\sigma}\right)$ then since $\eta$ is a top-dimensional simplex in $L_{\sigma}$ we have $\sigma \subseteq \eta$ let $i_{0}, \ldots, i_{p} \in\{0, \ldots, m\}$ denote the indices of the corresponding vertices of $\sigma$ in $\eta$. Let us write $\tau$ as $\tau=\left[b_{0}^{0}, \ldots b_{q_{0}}^{0}|\ldots| b_{0}^{m}, \ldots, b_{q_{m}}^{m}\right]$, where the $i$ 'th block, $\left|b_{0}^{i}, \ldots, b_{q_{i}}^{i}\right|$, lies over the $i$ 'th vertex in $\eta$. For $0 \leq s_{j} \leq q_{i_{j}}$ we define

$$
\tau^{s_{0} \cdots s_{p}}=\left[b_{0}^{i_{0}}, \ldots, b_{s_{0}}^{i_{0}}|\ldots| b_{0}^{i_{p}}, \ldots, b_{s_{p}}^{i_{p}}\right]
$$

and

$$
\tau_{s_{0} \cdots s_{p}}=\left[b_{0}^{0}, \ldots, b_{q_{0}}^{0}|\ldots| b_{s_{j}}^{i_{j}}, \ldots, b_{q_{i_{j}}}^{i_{j}}|\ldots| b_{0}^{m}, \ldots, b_{q_{m}}^{m}\right]
$$

then our map is given by

$$
\operatorname{AW}(\tau)=\sum_{0 \leq s_{j} \leq q_{i_{j}}} \tau^{s_{0} \cdots s_{p}} \otimes \tau_{s_{0} \cdots s_{p}}
$$

That is an Alexander-Whitney type map with respect to each block of vertices in $\tau$ lying over a vertex in $\sigma$.

The following lemma is a straightforward computation similar to the proof of the usual AW-map being a chain map.

LEMMA 5.5. The map

$$
\mathrm{AW}: P C_{k, m}\left(K / L_{\sigma}\right) \rightarrow \bigoplus_{k_{1}+k_{2}=k} P C_{k_{1}, p}(K / \sigma) \otimes P C_{k_{2}, m}\left(K / L_{\sigma}\right)
$$

is a chain map with respect to the boundary map $\partial_{F}$ from example 3.1 , that is

$$
\mathrm{AW} \partial_{F}=\partial_{F} \mathrm{AW}
$$


We have to specify $\int_{K / L} \omega \in \Omega^{*}(|N L|)$ as a form on $\Delta^{p} \times \eta$ for $\eta \in L_{\sigma}$. If for the moment we let $\eta$ be an $m$-simplex the formula is quite simple.

First pick an orientation of $\eta$, since the fibers of $\pi$ is oriented, this gives us an orientation of $Y_{\mid \eta}$ and hence a fundamental class $\left[Y_{\mid \eta}\right] \in P C_{n+m}(K / \sigma)$.

Now consider $N K_{\mid \pi^{-1}\left(\left|L_{\sigma}\right|\right)}$ as a subset of $|K|_{\mid \sigma} \times|K|_{|| L_{\sigma} \mid}$. We will define $\int_{K / L} \omega_{\mid \Delta^{p} \times \eta}$ by restricting $\omega$ to $\mathrm{AW}\left(\left[Y_{\mid \eta}\right]\right)$ and integrate along the fiber over $\Delta^{p} \times \eta$.

Set $s=\sum_{i=0}^{p} s_{i}$, then our formula will be given by

$$
\int_{K / L} \omega_{\mid \Delta^{p} \times \eta}=\sum_{\tau \in P S_{n, m}(K / \eta)} \sum_{0 \leq s_{j} \leq q_{i_{j}}} \varepsilon(\tau) \int_{\Delta^{p+s} \times \tau_{s_{0}, \ldots s_{p}} / \Delta^{p} \times \eta} \omega_{\tau^{s 0} \ldots \ldots p_{p}}^{(p+s)},
$$

where $\omega_{\tau^{s_{0} \ldots s_{p}}}^{(p+s)} \in \Omega^{*+n}\left(\Delta^{p} \times K_{\tau^{s_{0} \ldots s_{p}}}\right)$ and $\varepsilon(\tau)$ is the sign of $\tau$ in $\left[Y_{\mid \eta}\right]$. The integration shall be understood as follows: We restrict $\omega$ to $\Delta^{p+s} \times \tau_{s_{0} \ldots s_{p}}$ and then integrate it along the fibers over $\Delta^{p} \times \eta$ with respect to the map $\Delta^{p+s} \rightarrow \Delta^{p}$ given by

$$
\left(t_{0}, \ldots t_{p+s}\right) \mapsto\left(\sum_{i=0}^{s_{0}} t_{i}, \sum_{i=s_{0}+1}^{s_{0}+s_{1}+1} t_{i}, \ldots, \sum_{i=s_{0}+\ldots+s_{p-1}+p}^{s_{0}+\ldots+s_{p}+p} t_{i}\right)
$$

and the map $\tau_{s_{0} \ldots s_{p}} \rightarrow \eta$ which is just the restriction of $\pi$.

REMARK 5.7. In the above, we could also have chosen to pull $\omega$ back to $\Omega^{*+n}\left(\left|P N K / L_{\sigma}\right|\right)$ with $\ell$ and then integrate with respect to the map

$$
\Delta^{p} \times \Delta^{s_{0} \ldots s_{p}} \times \tau_{s_{0} \ldots s_{p}} \rightarrow \Delta^{p} \times \eta
$$

This gives the same result, but will be more convenient when we shall see that the two approaches to integration give the same result.

We still need to define the integral on $\Delta^{p} \times \eta^{\prime}$ for $\eta^{\prime} \in S_{k}\left(L_{\sigma}\right)$ a lowerdimensional simplex. This will actually just be the restriction of the integral on $\Delta^{p} \times \eta$ for $\eta$ a top-simplex such that $\eta^{\prime} \subseteq \eta$, we shall see that this is independent of which top-simplex we choose (this also shows that the resulting form is really simplicial on $\left.\left|L_{\sigma}\right|\right)$.

Let us first take a look at what happens to the formula (5.6) when the integral is restricted to $\Delta^{p} \times \eta^{\prime} \subseteq \Delta^{p} \times \eta$.

For a $\tau \in P S_{n, m}(K / \eta)$ we see that

$$
\int_{\Delta^{p+s} \times \tau_{s_{0} \ldots s_{p}} / \Delta^{p} \times \eta} \omega_{\tau^{s 0 \ldots s p}}^{(p+s)}
$$


restricted to $\Delta^{p} \times \eta^{\prime}$ is non-zero exactly when $\tau_{s_{0} \ldots s_{p}} \cap \pi^{-1}\left(\eta^{\prime}\right)$ and $\tau_{s_{0} \ldots s_{p}}$ have the same dimension $r=n-s$ in the direction of the fiber. That is $\tau_{s_{0} \ldots s_{p}} \cap \pi^{-1}\left(\eta^{\prime}\right) \in P S_{r, k}\left(K / \eta^{\prime}\right)$ and $\tau_{s_{0} \ldots s_{p}} \in P S_{r, m}(K / \eta)$ (the dimension in the direction of the fiber for a simplex in $K$ is given $\operatorname{asim}_{F} \tau=\operatorname{dim} \tau-\operatorname{dim} \pi(\tau)$ ).

Now in this case let $\alpha$ be the simplex in $L_{\sigma}$ 'spanned' by $\eta^{\prime}$ and $\sigma$, then $\tau \cap \pi^{-1}(\alpha)$ is $n$-dimensional in the fiber direction, so over each $\tilde{\eta} \in S_{m}\left(L_{\sigma}\right)$, with $\eta^{\prime} \subseteq \tilde{\eta}$, there is exactly one $\tilde{\tau} \in P S_{n, m}(K / \tilde{\eta})$ with $\tau \cap \pi^{-1}(\alpha) \subseteq \tilde{\tau}$ and in the expression (5.8) it would make no difference if we used $\tilde{\tau}$ instead of $\tau$.

We can also give an explicit formula in this case, but first we need some notation. For a top-simplex $\mu \in P S_{n, m}\left(K / L_{\sigma}\right)$ set $\tilde{\mu}=\mu \cap \pi^{-1}(\sigma)$. For a simplex $\rho \in P S_{r, k}\left(K / \eta^{\prime}\right)$ let

$F \rho=$

$\left\{\mu \in P S_{n, m}\left(K / L_{\sigma}\right) \mid \rho=\mu \cap \pi^{-1}\left(\eta^{\prime}\right), \operatorname{dim}_{F} \tilde{\mu}+\operatorname{dim}_{F} \rho-\operatorname{dim}_{F}(\tilde{\mu} \cap \rho)=n\right\}$.

Now write

$$
\rho=\left[c_{0}^{0}, \ldots, c_{r_{0}}^{0}|\ldots| c_{0}^{k}, \ldots, c_{r_{k}}^{k}\right] \text { and } \tilde{\mu}=\left[b_{0}^{0}, \ldots, b_{q_{0}}^{0}|\ldots| b_{0}^{p}, \ldots, b_{q_{p}}^{p}\right]
$$

with $\mu \in F \rho$ and let $i_{0}, \ldots, i_{l} \in\{0, \ldots, p\}$ and $j_{0}, \ldots, j_{l} \in\{0, \ldots, k\}$ denote the coinciding blocks in $\tilde{\mu}$ and $\rho$, that is

$$
\tilde{\mu} \cap \rho=\left[b_{0}^{i_{0}}, \ldots, b_{q_{i_{0}}}^{i_{0}}|\ldots| b_{0}^{i_{l}}, \ldots, b_{q_{i_{l}}}^{i_{l}}\right]=\left[c_{0}^{j_{0}}, \ldots, c_{q_{j_{0}}}^{j_{0}}|\ldots| c_{0}^{j_{l}}, \ldots, c_{q_{j_{l}}}^{j_{l}}\right] .
$$

As before we set

$$
\rho_{s_{0} \ldots s_{l}}=\left[c_{0}^{0}, \ldots, c_{r_{0}}^{0}|\ldots| c_{s_{v}}^{j_{v}}, \ldots, c_{r_{j_{v}}}^{j_{v}}|\ldots| c_{0}^{k}, \ldots, c_{r_{k}}^{k}\right]
$$

and

$$
\tilde{\mu}^{s_{0} \ldots s_{l}}=\left[b_{0}^{0}, \ldots, b_{q_{0}}^{0}|\ldots| b_{0}^{i_{v}}, \ldots, b_{s_{v}}^{i_{v}}|\ldots| b_{0}^{p}, \ldots, b_{q_{p}}^{p}\right]
$$

and then finally the integration formula is given on $\Delta^{p} \times \eta^{\prime}$ by

$$
\sum_{\rho \in P S_{*, k}\left(K / \eta^{\prime}\right)} \sum_{\{\tilde{\mu} \mid \mu \in F \rho\}} \sum_{0 \leq s_{\nu} \leq q_{i_{v}}} \varepsilon(\mu) \int_{\Delta^{p+s} \times \rho_{s_{0}, \cdots l} / \Delta^{p} \times \eta} \omega_{\tilde{\mu}^{s_{0} \cdots s_{l}}}^{(p+s)}
$$

THEOREM 5.10. 1. Let $\omega \in \Omega^{*+n}(|N K|)$ be a piecewise smooth normal simplicial form; then $\int_{K / L} \omega$ is a well-defined piecewise smooth normal simplicial form.

2. Let $\omega \in \Omega^{k+n-1}(|N K|)$, then we have a Stokes' theorem

$$
\int_{K / L} d \omega=\int_{\partial_{F} K / L} \omega+(-1)^{n} d \int_{K / L} \omega
$$


3. If $\partial Y=\emptyset$ then the map $\int_{K / L}: \Omega^{*+n}(|N K|) \rightarrow \Omega^{*}(|N L|)$ takes integral forms to integral forms and it induces a map $\pi_{!}: H_{\mathscr{D}}^{*+n}(Y, \mathrm{Z}) \rightarrow H_{\mathscr{D}}^{*}(Z, \mathrm{Z})$ in smooth Deligne cohomology.

Proof. 1. This follows at once from the construction.

2. First we observe that for $\omega \in \Omega^{k+n-1}(|N K|)$ we have on $\Delta^{p} \times \eta\left(\eta \in L_{\sigma}^{(n)}\right)$

$$
\begin{aligned}
& \int_{K / L} d \omega=\sum_{\tau \in P S_{n, m}(K / \eta)} \sum_{0 \leq s_{j} \leq q_{i_{j}}} \int_{\Delta^{p+s} \times \tau_{s_{0} \ldots p_{p}} / \Delta^{p} \times \eta}(d \omega)^{\tau^{s 0 \ldots s_{p}}} \\
& =\sum_{\tau \in P S_{n, m}(K / \eta)} \sum_{0 \leq s_{j} \leq q_{i_{j}}} \int_{\partial_{F}\left(\Delta^{p+s} \times \tau_{s_{0}, \ldots s_{p}}\right) / \Delta^{p} \times \eta} \omega_{\tau^{s_{0} \ldots s_{p}}}^{(+s)}+ \\
& +(-1)^{n} \sum_{\tau \in P S_{n, m}(K / \eta)} \sum_{0 \leq s_{j} \leq q_{i_{j}}} d \int_{\Delta^{p+s} \times \tau_{s_{0} \ldots s_{p}} / \Delta^{p} \times \eta} \omega_{\tau^{s_{0} \cdots s_{p}}}^{(p+s)} \\
& =\sum_{\tau \in P S_{n, m}(K / \eta)} \sum_{0 \leq s_{j} \leq q_{i_{j}}} \int_{\partial_{F}\left(\Delta^{p+s} \times \tau_{s_{0} \ldots s_{p}}\right) / \Delta^{p} \times \eta} \omega_{\tau^{s} 0 . s_{p}}^{(p+s)}+ \\
& +(-1)^{n} d \int_{K / L} \omega .
\end{aligned}
$$

In this formula, we recognize the first terms as $\int_{\partial_{F} K / L} \omega$ since lemma 5.5 gives us that $\partial_{F} A W\left(\left[Y_{\mid \eta}\right]\right)=A W\left(\partial_{F}\left[Y_{\mid \eta}\right]\right)$. Hence we have verified the formula for $\eta$ a top-dimensional simplex, and since the value of the integral on the other simplices is given by restrictions, the formula holds in general.

3. If $\omega \in \Omega^{*+n}(|N K|)$ is integral, then we observe that the only non-zero terms in (5.6) are those for $s=n$, that is, the integration is only with respect to the map $\Delta^{p+n} \rightarrow \Delta^{p}$, and the resulting forms are then clearly integral. We also see that there is a result similar to lemma 4.3, so it is now clear that we have an induced map in Deligne cohomology.

Now we are ready to compare the two integration maps. This comparison will also quite easily show that the first, smooth version of the integration map also takes integral forms to integral forms.

First, choose a triangulation of the fiber bundle $Y \rightarrow Z$ and let $\mathscr{V}=\left\{V_{j}\right\}_{j \in J}$ and $\mathscr{U}=\left\{U_{i}\right\}_{i \in I}$ be the associated coverings by the stars. Now let $K$ and $L$ be subdivisions of these triangulations so that every closed star of $K$ and $L$ lies inside an open set of $\mathscr{V}$ and $\mathscr{U}$ respectively. Then we get maps

$$
T: \Omega^{*}(|P N \mathscr{V} / \mathscr{U}|) \rightarrow \Omega^{*}(|P N K / L|), \quad T^{\prime}: \Omega^{*}(|N \mathscr{U}|) \rightarrow \Omega^{*}(|N L|)
$$

as above, inducing isomorphisms in cohomology. 
Lemma 5.11. If $\partial Y=\emptyset$ then the map $\int_{[Y / Z]}: \Omega^{*+n}(|N \mathscr{V}|) \rightarrow \Omega^{*}(|N \mathscr{U}|)$ takes integral forms to integral forms and hence induces a map in smooth Deligne cohomology.

Proof. In the following, we will make use of remark 5.7, that is, we will look at the integration map in terms of the prism complex.

Let $\beta \in \Omega^{k+n}(|N \mathscr{V}|)$ be an integral form. Now remark 4.1 ensures that the pull back $\ell^{*} \beta \in \Omega^{k+n}(|P N \mathscr{V} / \mathscr{U}|)$ lies in the subcomplex $\bigoplus_{q \leq n} \Omega^{k+n-q, q, 0}$ $(|P N \mathscr{V} / \mathscr{U}|)$. Note also that everything besides the term in $\Omega^{k, n, 0}(|P N \mathscr{V} / \mathscr{U}|)$ maps to zero under the integration map. Now the diagram

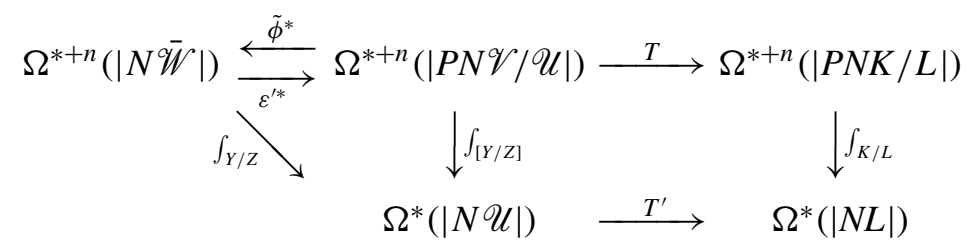

where the commutativity of the triangle and the outer square impliy that $\int_{K / L} T \varepsilon^{*}=T^{\prime} \int_{Y / Z}$. Furthermore, if we put $\beta^{\prime}=\varepsilon^{\prime *} \tilde{\phi}^{*} \beta$ then by corollary 3.6 we have

$$
\beta^{\prime}-\beta=h d \beta+d h \beta,
$$

where $h$ is the homotopy operator inducing the chain homotopy $\varepsilon^{*} \tilde{\phi}^{*} \sim$ id. Note that since $\tilde{\phi} \circ \varepsilon^{\prime}$ is the identity in the variable of the first simplex and in those on the nerve, $h$ maps $\Omega^{p, q, r}(|P N \mathscr{V} / \mathscr{U}|)$ into

$$
\bigoplus_{q^{\prime}<q} \Omega^{p, q-q^{\prime}-1, r+q^{\prime}}(|P N \mathscr{V} / \mathscr{U}|)
$$

so the image of $h$ maps to zero under the integration map. Now by definition we have

$$
\int_{[Y / Z]} \beta=\int_{Y / Z} \tilde{\phi}^{*} \beta=\int_{[Y / Z]} \beta^{\prime}
$$

and hence commutativity of the outer square in (5.12) gives

$$
T^{\prime} \int_{Y / Z} \tilde{\phi}^{*} \beta=\int_{K / L} T \varepsilon^{*} \tilde{\phi}^{*} \beta=\int_{K / L} T \beta^{\prime}=\int_{K / L} T \beta+\int_{K / L} T(h d \beta+d h \beta) .
$$

Also, since $\int_{K / L} T d h \beta=(-1)^{n-1} d \int_{K / L} T h \beta$ the last integral is zero. We therefore finally get

$$
T^{\prime} \int_{[Y / Z]} \beta=\int_{K / L} T \beta
$$

and since the right side is clearly integral, as noted above, we conclude that $\int_{[Y / Z]}$ maps integral forms to integral forms. 
THEOREM 5.13. If $\partial Y=\emptyset$ then the maps

$$
\int_{[Y / Z]}: \Omega^{*+n}(|N \mathscr{V}|) \rightarrow \Omega^{*}(|N \mathscr{U}|)
$$

and

$$
\int_{K / L}: \Omega^{*+n}(|N K|) \rightarrow \Omega^{*}(|N L|)
$$

induce the same map

$$
\pi_{!}: H_{\mathscr{D}}^{*+n}(Y, \mathrm{Z}) \rightarrow H_{\mathscr{D}}^{*}(Z, \mathrm{Z})
$$

in smooth Deligne cohomology.

Proof. This is similar to the proof above. Taking $\omega \in \Omega^{*+n}(|P N \mathscr{V} / \mathscr{U}|)$ with $d \omega=\varepsilon^{*} \alpha-\beta$, we set $\omega^{\prime}=\varepsilon^{*} \tilde{\phi}^{*} \omega$ and get $\omega^{\prime}-\omega=d h \omega+h d \omega$. As above we have

$$
\begin{aligned}
T^{\prime} \int_{[Y / Z]} \omega & =T^{\prime} \int_{[Y / Z]} \omega^{\prime}=\int_{K / L} T \omega^{\prime} \\
& =\int_{K / L} T \omega+d \int_{K / L} T h \omega+\int_{K / L} T h d \omega
\end{aligned}
$$

and, as in the proof of lemma 5.11 the last term vanishes, since $h d \omega=h \varepsilon^{*} \alpha-$ $h \beta=-h \beta$ because $\varepsilon^{\prime *} \tilde{\phi}^{*}$ obviously acts as the identity on $\varepsilon^{*} \alpha$. Hence we get

$$
T^{\prime} \int_{[Y / Z]} \omega=\int_{K / L} T \omega+d \tau
$$

where $\tau=\int_{K / L} T h \omega$.

Corollary 5.14 .

$$
\pi_{!}: H_{\mathscr{D}}^{*+n}(Y, \mathrm{Z}) \rightarrow H_{\mathscr{D}}^{*}(Z, \mathrm{Z})
$$

is independent of choice of coverings, partition of unity and triangulations. In particular this proves theorem 1.1.

Recall from example 3.1 that in the case of a fiber bundle $Y \rightarrow Z$ with compact oriented fibers, where $\partial Y \neq \emptyset$ and a given triangulation of the bundle $\partial Y \rightarrow Z$, it is possible to extend this triangulation to a triangulation of the bundle $Y \rightarrow Z$. We shall see that the integral is independent of this extension, thus proving theorem 1.2.

THEOREM 5.15. Given a form $\omega \in \Omega^{*+n}(|N \mathscr{V}|)$ representing a class in Deligne cohomology and a triangulation of $\partial Y \rightarrow Z$ compatible with the 
covering $\mathscr{V}$ and two extensions $\left|K_{1}\right| \rightarrow|L|$ and $\left|K_{2}\right| \rightarrow|L|$ of this to $Y \rightarrow Z$ then

$$
\int_{K_{1} / L} T_{1} \omega \sim \int_{K_{2} / L} T_{2} \omega \text { in } \Omega^{*}(|N L|),
$$

where $T_{i}: \Omega^{*}(|N \mathscr{V}|) \rightarrow \Omega^{*}\left(\left|N K_{i}\right|\right), i=1,2$ are given as above.

Proof. As in the proof of theorem 5.13 we have a $\omega^{\prime} \in \Omega^{*+n}(|P N \mathscr{V} / \mathscr{U}|)$ so that $\omega^{\prime}=\omega+d h \omega+h d \omega$, and we get, for $i=1,2$,

$$
\begin{aligned}
T^{\prime} \int_{[Y / Z]} \omega & =T^{\prime} \int_{[Y / Z]} \omega^{\prime}=\int_{K_{i} / L} T_{i} \omega^{\prime} \\
& =\int_{K_{i} / L} T_{i} \omega+\int_{K_{i} / L} T_{i}(d h \omega+h d \omega) \\
& =\int_{K_{i} / L} T_{i} \omega+\int_{K_{i} / L} T_{i} d h \omega
\end{aligned}
$$

where $T^{\prime}: \Omega^{*}(|N \mathscr{U}|) \rightarrow \Omega^{*}(|N L|)$. Now the theorem follows from the fact that

$$
\int_{K_{i} / L} T_{i} d h \omega=d \int_{K_{i} / L} T_{i} h \omega \pm \int_{\partial_{F} K_{i} / L} T_{i} h \omega,
$$

where the last term is easily seen to be independent of $i$.

\section{Products}

The smooth Deligne cohomology groups comes with a product structure which has a quite simple description in the Čech-de Rham complex (see [1, ch. 1] for a detailed description).

In this section we define a product in $\Omega^{*}(|N \mathscr{U}|)$ compatible with this. This product is well behaved on the chain level with respect to the integration map in Deligne cohomology. Note that this product is different from (although chain homotopic to) the usual wedge product in $\Omega^{*}(|N \mathscr{U}|)$ which does not preserve the set of integral forms and hence does not induce a map in Deligne cohomology.

First consider the maps

$$
\pi_{i}:\left|P_{1} N \mathscr{U}\right| \rightarrow|N \mathscr{U}|, \quad i=1,2
$$

where

$$
\pi_{1}: \Delta^{q_{0} q_{1}} \times U_{i_{0} \ldots i_{q_{0}+q_{1}+1}} \rightarrow \Delta^{q_{0}} \times U_{i_{0} \ldots i_{q_{0}}}
$$

is given by

$$
\left(r^{0}, r^{1}, x\right) \mapsto\left(r^{0}, x\right)
$$


and similarly

$$
\pi_{2}: \Delta^{q_{0} q_{1}} \times U_{i_{0} \ldots i_{q_{0}+q_{1}+1}} \rightarrow \Delta^{q_{1}} \times U_{i_{q_{0}+1} \ldots i_{q_{0}+q_{1}+1}}
$$

is given by

$$
\left(r^{0}, r^{1}, x\right) \mapsto\left(r^{1}, x\right)
$$

For $t \in \Delta^{1}$ we have the map

$$
\ell_{t}:\left|P_{1} N \mathscr{U}\right| \rightarrow|N \mathscr{U}|
$$

given by

$$
\ell_{t}\left(r^{0}, r^{1}, x\right)=\left(t r^{0},(1-t) r^{1}, x\right)
$$

It is clearly a homeomorphism for $t \in \stackrel{\circ}{\Delta}^{1}$.

The inverse is given as follows. Take an $\left(r_{0}, \ldots, r_{n}, x\right) \in \Delta^{n} \times U_{i_{0} \ldots i_{n}}$ and choose $p$ so that $\sum_{i=0}^{p-1} r_{i} \leq t<\sum_{i=0}^{p} r_{i}$. Then

$$
\begin{aligned}
\ell_{t}^{-1}\left(r_{0}, \ldots, r_{n}, x\right)=\left(\left(\frac{r_{0}}{t}, \ldots,\right.\right. & \left.\frac{r_{p-1}}{t}, 1-\frac{\sum_{i=0}^{p-1} r_{i}}{t}\right), \\
& \left.\left(1-\frac{\sum_{i=p+1}^{n} r_{i}}{1-t}, \frac{r_{p+1}}{1-t}, \ldots, \frac{r_{n}}{1-t}\right), s_{p} x\right) .
\end{aligned}
$$

Choose a smooth bump-function $\phi: \mathrm{R} \rightarrow \mathrm{R}$ so that the following holds

1. $\int_{0}^{1} \phi(t) d t=1$.

2. $\lim _{t \rightarrow 0} \phi(t) / t^{p}=0$ and $\lim _{t \rightarrow 1} \phi(t) /(1-t)^{p}=0$ for all $p \in \mathbf{N}$. We now have

DeFinition 6.1. The product

$$
\wedge_{1}: \Omega^{*}(|N \mathscr{U}|) \times \Omega^{*}(|N \mathscr{U}|) \rightarrow \Omega^{*}(|N \mathscr{U}|) .
$$

is given by

$$
\omega_{1} \wedge_{1} \omega_{2}:=\int_{\Delta^{1}} \phi(t) d t \wedge\left(\ell_{t}^{-1}\right)^{*}\left(\pi_{1}^{*} \omega_{1} \wedge \pi_{2}^{*} \omega_{2}\right)
$$

The choice of bump-function ensures that there are no convergence problem. So this is well-defined and seen to give a normal simplicial form.

Most of the following proposition is trivial.

Proposition 6.2. 1. Two different choices of bump-function give chainhomotopic products. 
2. For $\omega_{1} \in \Omega^{p}(|N \mathscr{U}|)$ and $\omega_{2} \in \Omega^{q}(|N \mathscr{U}|)$ we have $d\left(\omega_{1} \wedge_{1} \omega_{2}\right)=$ $d \omega_{1} \wedge_{1} \omega_{2}+(-1)^{p} \omega_{1} \wedge_{1} d \omega_{2}$.

3. $I_{\Delta}: \Omega^{*}(|N \mathscr{U}|) \rightarrow \check{\Omega}^{*}(\mathscr{U})$ is multiplicative.

4. If $\omega_{1}, \omega_{2} \in \Omega_{\mathrm{Z}}^{*}(|N \mathscr{U}|)$ then $\omega_{1} \wedge_{1} \omega_{2} \in \Omega_{\mathrm{Z}}^{*}(|N \mathscr{U}|)$.

Proof. 1. This is trivial since two choices of bump-functions that satisfy the required conditions are certainly homotopic by a linear homotopy through such bump-functions.

2. Follows from the corresponding formula for the wedge product.

3. Suppose $\omega_{1} \wedge_{1} \omega_{2} \in \Omega^{n, m}(|N \mathscr{U}|)$ then we have

$$
\begin{aligned}
\int_{\Delta^{n}}\left(\omega_{1} \wedge{ }_{1} \omega_{2}\right)_{i_{0} \ldots i_{n}} \\
=\int_{\Delta^{n}} \int_{\Delta^{1}} \phi(t) d t \wedge\left(\ell_{t}^{-1 *}\left(\pi_{1}^{*} \omega_{1} \wedge \pi_{2}^{*} \omega_{2}\right)\right)_{i_{0} \ldots i_{n}} \\
=\int_{\Delta^{1} \times \Delta^{n}} \phi(t) d t \wedge\left(\ell_{t}^{-1 *}\left(\pi_{1}^{*} \omega_{1} \wedge \pi_{2}^{*} \omega_{2}\right)\right)_{i_{0} \ldots i_{n}} \\
=\sum_{p+q=n} \int_{\Delta^{1} \times \Delta^{p} \times \Delta^{q}} \phi(t) d t \wedge \ell_{t}^{*} \eta_{p}^{*}\left(\ell_{t}^{-1 * 1 *}\left(\pi_{1}^{*} \omega_{1} \wedge \pi_{2}^{*} \omega_{2}\right)\right)_{i_{0} \ldots i_{n}} \\
=\sum_{p+q=n} \int_{\Delta^{1} \times \Delta^{p} \times \Delta^{q}} \phi(t) d t \wedge\left(\omega_{1}\right)_{i_{0} \ldots i_{p}} \wedge\left(\omega_{2}\right)_{i_{p} \ldots i_{n}} \\
=\sum_{p+q=n} \int_{\Delta^{p}}\left(\omega_{1}\right)_{i_{0} \ldots i_{p}} \wedge \int_{\Delta^{q}}\left(\omega_{2}\right)_{i_{p} \ldots i_{n} .} .
\end{aligned}
$$

So $I\left(\omega_{1} \wedge_{1} \omega_{2}\right)=I\left(\omega_{1}\right) \wedge I\left(\omega_{2}\right)$ as claimed.

4. This follows directly from the proof of 3 .

REMARK 6.3. Unfortunately the product is neither commutative nor associative on the chain level but 3 above ensures us that it is up to chain homotopy.

The product is well-behaved with respect to the integration map in section 4 .

Proposition 6.4. For $\omega_{1} \in \Omega^{p+n}(|N \mathscr{V}|)$ and $\omega_{2} \in \Omega^{q}(|N \mathscr{U}|)$ we have

$$
\left(\int_{[Y / Z]} \omega_{1}\right) \wedge_{1} \omega_{2}=\int_{[Y / Z]} \omega_{1} \wedge_{1} \pi^{*} \omega_{2}
$$

Proof. Note that

$$
\left(\pi_{1} \times \pi_{2}\right) \circ \ell_{t}^{-1} \circ \ell \circ \tilde{\phi}=\left(\ell \circ \tilde{\phi} \circ \pi_{1} \times \ell \circ \tilde{\phi} \circ \pi_{2}\right) \circ \ell_{t}^{-1}:|N \mathscr{W}| \rightarrow|N \mathscr{V}| \times|N \mathscr{V}|
$$


So for a pair of forms $\omega, \tau \in \Omega^{*}(|N \mathscr{U}|)$ we get the relation

$$
(\ell \circ \tilde{\phi})^{*}\left(\omega \wedge_{1} \tau\right)=(\ell \circ \tilde{\phi})^{*} \omega \wedge_{1}(\ell \circ \tilde{\phi})^{*} \tau .
$$

This implies that

$$
\begin{aligned}
\int_{[Y / Z]} \omega_{1} \wedge_{1} \pi^{*} \omega_{2} & =\int_{Y / Z}(\ell \circ \tilde{\phi})^{*} \omega_{1} \wedge_{1}(\ell \circ \tilde{\phi})^{*} \pi^{*} \omega_{2} \\
& =\int_{Y / Z}(\ell \circ \tilde{\phi})^{*} \omega_{1} \wedge_{1} \pi^{*} \omega_{2} \\
& =\left(\int_{Y / Z}(\ell \circ \tilde{\phi})^{*} \omega_{1}\right) \wedge_{1} \omega_{2} \\
& =\left(\int_{[Y / Z]} \omega_{1}\right) \wedge_{1} \omega_{2}
\end{aligned}
$$

as stated above.

Let us move on to Deligne cohomology where the product structure is a little different.

Definition 6.6. Let $\omega_{1} \in \Omega^{p}(|N \mathscr{U}|)$ and $\omega_{2} \in \Omega^{q}(|N \mathscr{U}|)$ be two forms representing classes in Deligne cohomology. That is $d \omega_{i}=\varepsilon^{*} \alpha_{i}-\beta_{i}$, where $\alpha_{i}$ is a global form and $\beta_{i}$ is integral. Then we define

$$
\omega_{1} \tilde{\wedge} \omega_{2}:=\omega_{1} \wedge_{1} \varepsilon^{*} \alpha_{2}+(-1)^{p+1} \beta_{1} \wedge_{1} \omega_{2}
$$

Some calculations show that this induces a well-defined product in Deligne cohomology. E.g. take another representative $\omega_{1}+\beta$ for the class $\left[\omega_{1}\right]$ then we have

$$
\begin{aligned}
\left(\omega_{1}+\beta\right) \tilde{\wedge} \omega_{2} & =\left(\omega_{1}+\beta\right) \wedge_{1} \varepsilon^{*} \alpha_{2}+(-1)^{p+1}\left(\beta_{1}+d \beta\right) \wedge_{1} \omega_{2} \\
& =\omega_{1} \tilde{\wedge} \omega_{2}+\beta \wedge_{1} \varepsilon^{*} \alpha_{2}+(-1)^{p+1} d \beta \wedge_{1} \omega_{2} \\
& =\omega_{1} \tilde{\wedge} \omega_{2}+d\left(\beta \wedge_{1} \omega_{2}\right)+\beta \wedge_{1} \beta_{2} \\
& \sim \omega_{1} \tilde{\wedge} \omega_{2} .
\end{aligned}
$$

Notably $d\left(\omega_{1} \tilde{\wedge} \omega_{2}\right)=\varepsilon^{*}\left(\alpha_{1} \wedge_{1} \alpha_{2}\right)-\beta_{1} \wedge_{1} \beta_{2}$.

With this product on $\Omega^{*}(|N \mathscr{U}|)$, the map $I_{\Delta}$ of section 2.1 between the simplicial and the Čech-de Rham model for Deligne cohomology becomes an isomorphism of graded rings.

The next proposition follows directly from proposition 6.4. 
Proposition 6.7. For $\omega_{1} \in \Omega^{p+n}(|N \mathscr{V}|)$ and $\omega_{2} \in \Omega^{q}(|N \mathscr{U}|)$ representing classes in Deligne cohomology, we have

$$
\left(\int_{[Y / Z]} \omega_{1}\right) \tilde{\wedge} \omega_{2}=\int_{[Y / Z]} \omega_{1} \tilde{\wedge} \pi^{*} \omega_{2} .
$$

REMARK 6.9. The product described above simplifies proposition 5.17 in [5]. Since we can choose $\gamma_{1} \wedge_{1} \gamma_{2}$ as representative for $u_{1} \cup u_{2}$.

\section{REFERENCES}

1. Brylinski, J.-L., Loop Spaces, Characteristic Classes and Geometric Quantization, Progr. Math. 107, 1993.

2. Cheeger, J., and Simons, J., Differential characters and geometric invariants, in Geometry and Topology, Proc. Spec. Year, College Park/Md. 1983/84, eds. J. Alexander and J. Harper, 50-80, Lecture Notes in Math. 1167 (1985).

3. Dupont, J. L., Simplicial de Rham cohomology and characteristic classes of flat bundles, Topology 15 (1976), 233-245.

4. Dupont, J. L., Curvature and characteristic classes, Lecture Notes in Math. 640 (1978).

5. Dupont, J. L., and Kamber, F. W., Gerbes, simplicial forms and invariants for families of foliated bundles, Comm. Math. Phys. 253 (2005), 253-282.

6. Freed, D., Classical Chern-Simons theory, part 2, Houston J. Math. 28 (2002), 293-310.

7. Gomi, K., and Terashima, Y., A fibre integration formula for the smooth Deligne cohomology, Internat. Math. Res. Notices 13 (2000), 699-708.

8. Hopkins, M. J., and Singer, I. M., Quadratic functions in geometry, topology, and M-theory, math.AT/0211216.

9. Johnson, F. E. A., On the triangulation of smooth fibre bundles, Fund. Math. 118 (1983), $39-58$.

10. Murray, M. K., and Stevenson, D., Bundle gerbes: stable isomorphism and local theory, J. London Math. Soc. (2) 62 (2000), 925-937.

11. Phillips, A. V., and Stone, D. A., The Chern-Simons character of a lattice gauge field, Quantum topology, 244-291, Ser. Knots Everything 3., World Sci. Publishing, River Edge, NJ, 1993.

DEPARTMENT OF MATHEMATICS

UNIVERSITY OF AARHUS

DK-8000 ÅRHUS

DENMARK

E-mail: dupont@imf.au.dk, runel@imf.au.dk 